\title{
Decoding key transient inter-catalyst interactions in a metallaphotoredox- catalysed cross-electrophile coupling reaction
}

\author{
Bart Limburg*, Àlex Cristòfol, Arjan W. Kleij* \\ anstitute of Chemical Research of Catalonia (ICIQ), The Barcelona Institute of Science and Technology, Av. Països Catalans 16, 43007 Tarragona \\ (Spain)
}

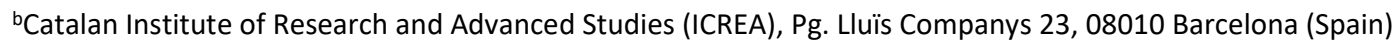

E-mail: blimburg@iciq.es, akleij@iciq.es

\begin{abstract}
Metallaphotoredox chemistry has recently witnessed a renaissance through the use of abundant first-row transition metals combined with suitable photocatalysts. The intricate details arising from the combination of two (or more) catalytic components during the reaction and specially the inter-catalyst interactions remain poorly understood. As a representative example of a catalytic process featuring such intricacies, we here present a meticulous study of the mechanism of a cobalt-organophotoredox catalysed allylation of aldehydes. Importantly, the commonly proposed elementary steps in reductive metallaphotoredox chemistry are more complex than previously assumed. After initial reductive quenching, a transient charge-transfer complex forms that interacts with both the transition-metal catalyst, as well as the catalytic base. Surprisingly, the former interaction leads to deactivation due to induced charge recombination, while the latter promotes deprotonation of the electron donor, which is a crucial step in order to promote productive catalysis, but is often neglected. Due to the low efficiency of this process, the overall catalytic reaction is photon-limited and the cobalt catalyst remains in a dual resting state awaiting photoinduced reduction. These new insights are of general importance to the synthetic community, as photoredox chemistry has become a powerful tool used in the creation of elusive compounds through carbon-carbon bond formations. Understanding the underlying factors that determine the efficiency of such reactions provides a conceptually stronger reactivity paradigm to empower future approaches to synthetic challenges that rely on dual metallaphotoredox catalysis.
\end{abstract}

Intro

Transition metal catalysis has been paramount in the development of stereoselective carbon-carbon bond formation reactions that are essential for the synthesis of natural products and novel pharmaceutical scaffolds. Historically, precious second- and third-row transition metals have been key catalyst components in such processes, since the reactivity and synthetic use of the more earth-abundant $3 \mathrm{~d}$ transition metals have been much harder to control and implement. Within the era of 'dual' catalytic metallaphotoredox catalysis, ${ }^{1-3}$ the utilisation of $3 \mathrm{~d}$ transition metal based catalysts has witnessed a remarkable revival and delivered new reactivity paradigms that are enabled through a delicate control over their available redox states allowing productively for single electron transfer (SET) pathways and radical-centred coupling reactions complementing the photoredox processes. 
In this context, dual catalytic cross-electrophile coupling reactions ${ }^{2,4-6}$ are of particular interest, as these strategies avoid the need to (pre)synthesise carbon nucleophiles. ${ }^{7}$ In such reactions, electrons are released from electron donors to in situ generated nucleophilic carbon species that engage in cross-coupling with carbon electrophiles forming a carbon-carbon (C-C) bond. While interest was initially focused on the combination of nickel or copper catalysis with photoredox catalysis, recently cobalt has emerged as a potent, alternative metal in dual catalytic procedures. ${ }^{8-10}$ The use of photoredox catalysis is beneficial as it avoids organometallic precursors and/or intermediates that are key to cross-electrophile coupling reactions by using suitable organic electron donors. This amplifies the functionality in the precursors and opposes the utilisation of stoichiometric reducing zerovalent metal additives (e.g., $\mathrm{Zn}$ or $\mathrm{Mn}$ ) which are generally less tolerant towards substrate diversity.,11

However, the presence of a second (or multiple) catalytic system complicates the mechanism of the reaction, ${ }^{12-16}$ and therefore a simplified model of the photoredox catalysis cycle is usually presented (Figure 1a), in which electrons are directly available for SET to the transition metal catalyst following reductive quenching. However, the nature of the interactions between the photoredox catalyst and the transition metal catalyst are likely more complex. ${ }^{12,16}$ In addition, photoredox catalytic reactions are often limited by the photon flux, and thus the usual rate-limiting steps for the transition metal driven cycle are unlikely to apply, which has important consequences for the overall kinetics of the reaction, and consequently, towards process scale up. In order for photocatalytic processes to become generic tools in synthetic organic chemistry, it is vital to gain a deeper understanding of how the catalytic systems are intertwined and uncover the mutual influence of the separate catalytic components in relation to the overall performance of the process.

While mechanistic studies for cobalt catalysis have been thoroughly probed under reductive conditions using chemical reductants, ${ }^{17-21}$ or electrochemically, ${ }^{22}$ it remains unknown whether dual metal/photoredox approaches proceed through the occurrence of similar intermediates. Several features of Co-catalysis under reductive conditions remain open for debate including the intermediacy of either $\mathrm{Co}^{\prime}$ or $\mathrm{Co}^{0}$-species ${ }^{11,22,23}$ and in dual $\mathrm{Co} /$ photoredox catalysis particularly how the effectiveness of different catalyst components and additives can be rationally optimised. A detailed knowledge of such multi-catalytic approaches offers a way to create new opportunities for various transformations that operate under similar mechanistic regimes thereby advancing this chemistry beyond empirical process optimisation.

Recently, our group and several others reported stereoselective cobalt-catalysed allylation of aldehydes as a promising new way to synthesise highly functional compounds through C-C bond formation. ${ }^{23-26}$ Such processes are mechanistically reminiscent to other important C-C bond formation reactions in synthetic chemistry, for which metallaphotoredox catalysis recently demonstrated to be a powerful methodology. ${ }^{9,10,27-31}$ Here, we describe a detailed mechanistic analysis of a stereoselective $\mathrm{C}-\mathrm{C}$ bond formation reaction that can be considered as a representative example for various types of reductive dual metallaphotoredox catalytic $\mathrm{C}-\mathrm{C}$ bond formation reactions, and we give special attention to the inter-catalyst interactions (Figure 1b). Specifically, we elucidated the full mechanism of the reductive allylation of aldehydes involving photoredox, cobalt and base catalysis (Figure 1c). Herein we show that the initially formed charge-separated state formed after excited-state SET is a key intermediate that 
interacts productively with the catalytic base, and counter-productively with the cobalt catalyst (Figure 1b). Furthermore, we demonstrate that the cobalt cycle is fast compared to the number of photons absorbed per unit time and therefore the reaction is limited by the light intensity and, unexpectedly, controlled by inter-catalyst interactions.

a)

$$
\text { commonly simplified }
$$
photocatalytic step

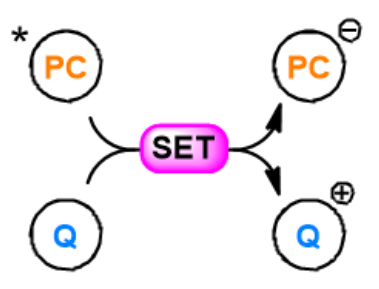

b)

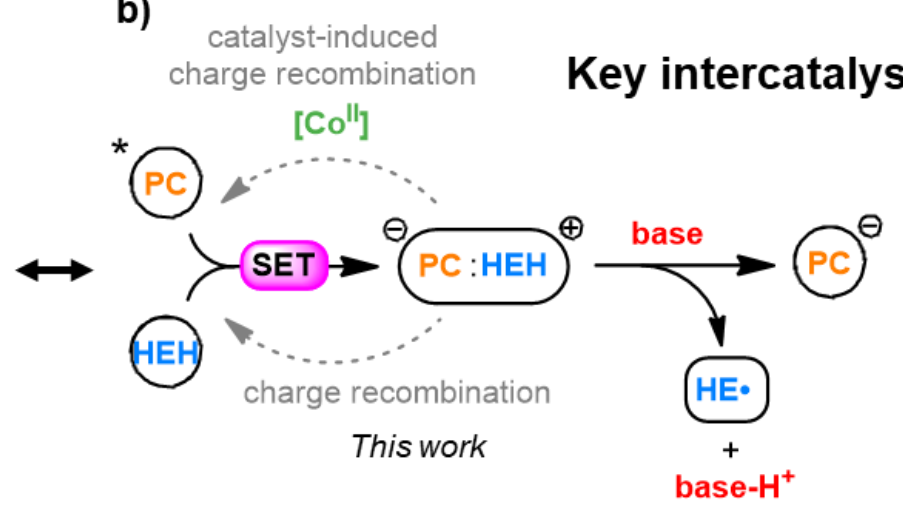

c) Representative metallaphotoredox catalysed C-C bond formation reaction:<smiles>C=C[C@@]1(c2ccccc2)COC(=O)O1</smiles>

1<smiles>[R]C1=C(C)NC(C)=C([R])C1[CH+]C(=O)c1ccccc1</smiles>

HEH<smiles>CC(=O)OC1Cc2ccc(C)cc2CC2C[C@@H](C)CCC2C1</smiles>

BIue LED T<smiles>[R]c1cc([R])c(C)nc1C</smiles>

3

9:1 syn:anti

Elucidated through:

- Transient absorption

- Electrochemistry

- In situ UV-vis

- Kinetic modeling

- Density functional theory

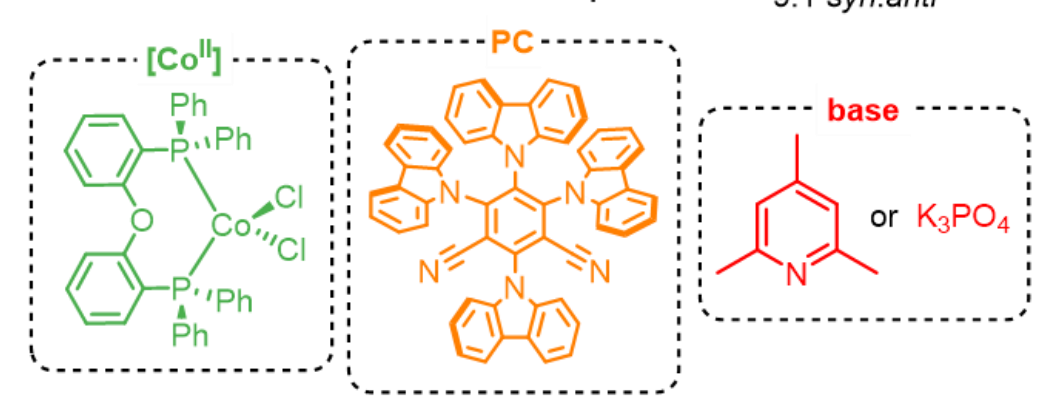

Figure 1. a) Commonly proposed single electron transfer (SET) step for reductive quenching, where an electron is transferred immediately from the quencher $(\mathrm{Q})$ to the photocatalyst $(\mathrm{PC})$, and the products $\left(\mathrm{Q}^{*^{+}}\right.$and $\left.\mathrm{PC} \mathrm{C}^{--}\right)$are considered solubilised. $\left.\mathrm{b}\right)$ Important transient inter-catalyst interactions determined in this work that determine the efficiency of the reaction. c) The representative metallaphotoredox catalytic crosselectrophile coupling process studied in this work.

\section{Results}

Kinetics. We started our investigations by studying the kinetics of the reaction presented in Figure $1 \mathrm{c}$ to determine the order of reagents and the rate-limiting step. Under optimised turnover conditions as described previously, ${ }^{26}$ using $\mathrm{K}_{3} \mathrm{PO}_{4}$ as a base, the disappearance of 1 was monitored by the evolution of its characteristic IR-band at $1815 \mathrm{~cm}^{-1}$ (Figure 2a). We first confirmed by NMR that the data obtained by IR corresponds to a 1:1 transformation of 1 to 3 without any observable intermediates (Figure 2b). A striking observation is that the reaction rate increases until $\mathbf{1}$ has been exhausted after which it necessarily comes to a sudden halt. This indicates, first, that the reaction is likely zero order in the substrates, and second, that a species that speeds up the reaction is formed over time. Initially we considered that a pre-activation of the catalyst was responsible for the acceleration of the reaction. However, as discussed below, the starting cobalt complex, [Co(DPEPhos) $\left.\mathrm{Cl}_{2}\right]$, is part of the catalytic cycle and therefore this cannot be the cause. By changing the base from $\mathrm{K}_{3} \mathrm{PO}_{4}$ to 2,4,6-collidine, we observed a higher initial rate and the reaction speed-up was less evident (Figure 2c). When the byproduct of the reaction, HEPy, is added as an additive, an 
intermediate rate is observed, whereas no acceleration is observed using product $\mathbf{3}$. Therefore, the reaction follows an autocatalytic pathway and its rate depends on the base and its concentration. Indeed, when kinetic data of the experiment with additionally added HEPy is offset to start at the end of the reaction using $\mathrm{K}_{3} \mathrm{PO}_{4}$, the curve appears to continue seamlessly (see Figure 2c), showing that the increased rate is due to formation of the base HEPy during the reaction. When plotting the quantum yield of the reaction as a function of the amount of HEPy produced (assuming a 1:1 production of HEPy from the consumption of $\mathbf{1}$ ), an increasing curve is produced, until the reaction is exhausted (i.e., full consumption of 1 ), see Figure $2 \mathrm{~d}$.

We then set out to determine the influence of the light intensity and the order of substrates $\mathbf{1}$ and $\mathbf{2}, \mathrm{HEH}$, and $\left[\mathrm{Co}(\mathrm{DPEPhos}) \mathrm{Cl}_{2}\right]\left(\left[\mathrm{CO}^{\prime \prime}\right]\right)$. Decreasing or increasing the light intensity speeds up or slows down the reaction, indicating that the reaction is photon-limited (see $\mathrm{SI}$ ). The initial quantum yield of the reaction using $\mathrm{K}_{3} \mathrm{PO}_{4}$ as base is $1.3 \%$, which can be increased to $7 \%$ by using collidine. The order in the substrates was further scrutinised by varying their concentrations. Neither diluting the mixture (effectively decreasing the concentration of all reagents), nor increasing the concentration of $\mathbf{1}, \mathbf{2}$, or increasing the scale leads to any notable difference in the kinetic trace (see SI). Therefore, $0^{\text {th }}$ order in all reagents is confirmed, caused by the photon-limited regime.

The concentration of [Co"], however, does affect the kinetics. Counterintuitively, increasing the concentration of [Co"] leads to a slower reaction, and vice versa (Figure $2 \mathrm{e}$ and $\mathrm{f}$ ). The effect is observed both when using $\mathrm{K}_{3} \mathrm{PO}_{4}$ or collidine as base. We initially considered that quenching of the excited state by [Co"] might be responsible, but quickly discarded this on basis of Stern-Volmer analysis (see SI), showing that [Co"] does not compete with HEH for quenching of the excited state under turnover conditions. Additionally, the cobalt complex does not absorb at the irradiation wavelength, so it does not compete with light absorption. Interestingly, the slower rate that results from doubling the concentration of [Co"] can be counteracted by doubling the concentration of base (see Figure 2f), indicating that a competition between [Co"] and the base exists in an intermediary step of the reaction mechanism. 


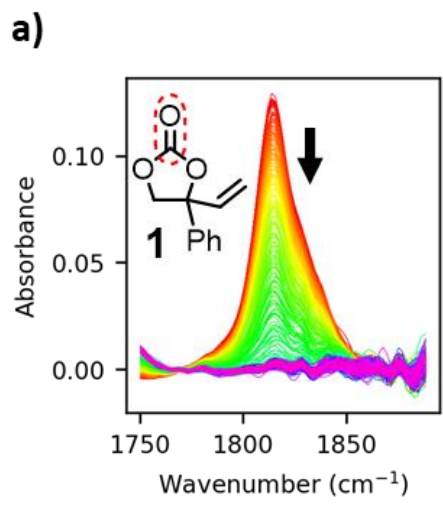

d)

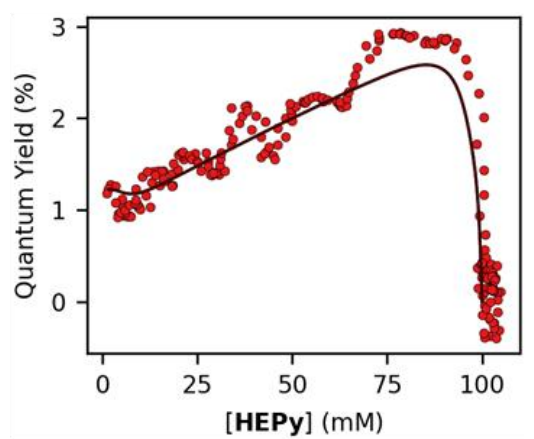

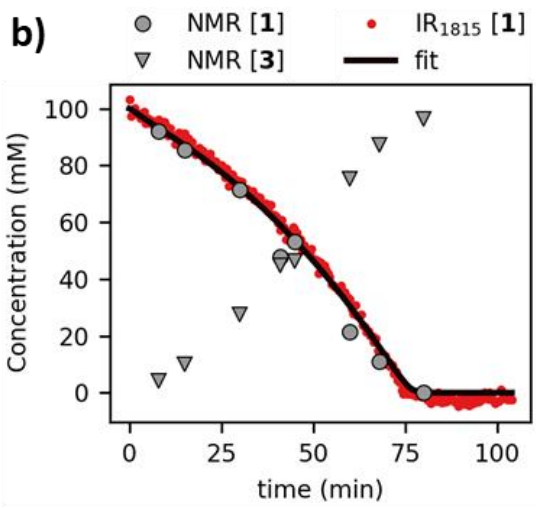

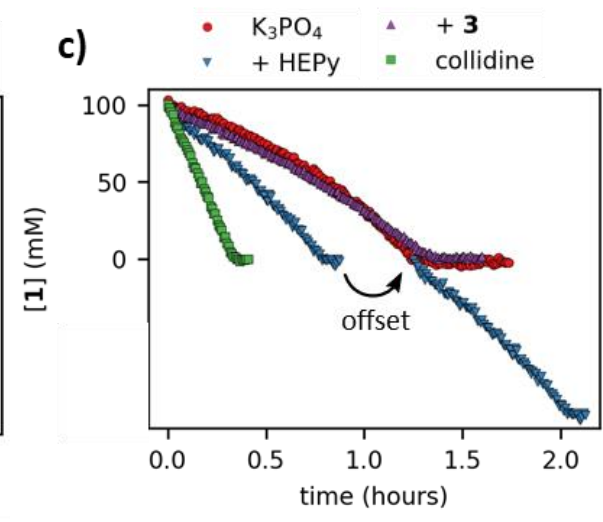

e)

$\mathrm{K}_{3} \mathrm{PO}$
$\mathrm{Col}$

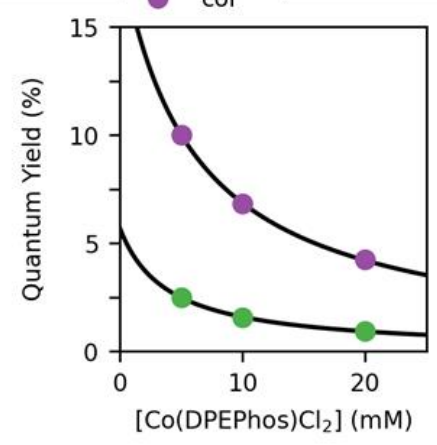

f)

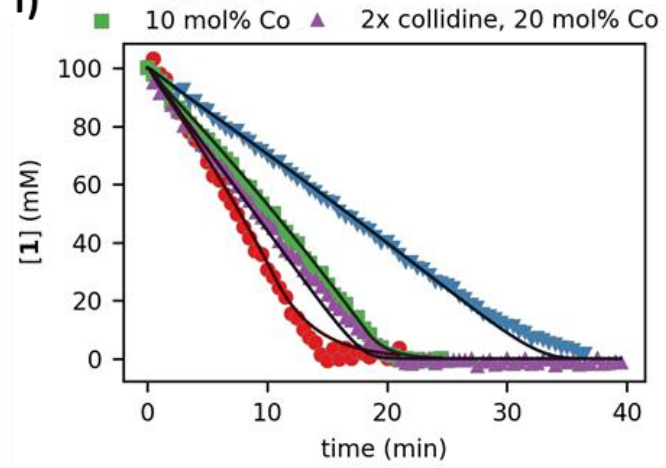

Figure 2. a) Evolution of the characteristic $\mathrm{C}=\mathrm{O} I \mathrm{IR}$ band at $1815 \mathrm{~cm}^{-1}$ during the reaction. b) Kinetic profile of the reaction when $\mathrm{K}_{3} \mathrm{PO}_{4}$ is $\mathrm{used}$ as a base. Conditions: $1(0.1 \mathrm{mmol}), 2(0.15 \mathrm{mmol}), \mathrm{HEH}(0.15 \mathrm{mmol})$, [Co"] $(10 \mu \mathrm{mol}), \mathrm{PC}(2 \mu \mathrm{mol}), \mathrm{K}_{3} \mathrm{PO}_{4}(0.15 \mathrm{mmol})$, THF ( $\left.1 \mathrm{~mL}\right)$, blue LED irradiation $\left(\lambda_{\mathrm{em}}=445 \mathrm{~nm}, 0.7 \mathrm{~A}, 1.2 \mu\right.$ einstein $\left.\mathrm{s}^{-1}\right)$. Red dots: data obtained through in situ monitoring the $\mathrm{C}=0$ IR stretch frequency of 1 at 1815 $\mathrm{cm}^{-1}$. Black line: fit to the kinetic model. Grey circles and grey triangles: remaining $\mathbf{1}$ and produced $\mathbf{3}$ as observed by ${ }^{1} \mathrm{H}$ NMR at different times with 1,3,5-mesitylene as internal standard. c) Kinetic profile in the presence of different bases. The blue and purple triangles are in the presence of HEPy or 3, respectively, in addition to $\mathrm{K}_{3} \mathrm{PO}_{4}$. The green squares are by substituting $\mathrm{K}_{3} \mathrm{PO}_{4}$ with 0.15 mmol 2,4,6-collidine. d) Quantum yield derived (using a Savitzky-Golay filter) from the kinetic trace in b) as a function of HEPy produced during the reaction to display the autocatalytic behaviour. The black line is the calculated quantum yield according to the kinetic model. e) Initial quantum yield of the reaction for the two bases as a function of the concentration of cobalt catalyst [Co"], fit to the equation $y=1 /(a+b x)$, a model for competitive kinetics from the intermediate ${ }^{3}\left[\mathrm{PC}^{\bullet-}: \mathrm{HEH}^{\bullet+}\right]$ (see $\mathrm{SI}$ ). f) Kinetic profiles of the reaction with 2,4,6-collidine as base and different concentrations of [Co"] . Solid lines: fits to the kinetic model.

Initial photoreactions. To study the competitive effect between base and [Co"], we investigated how the reductive equivalents required for the cross-electrophile reaction are produced. Due to the use of thermally activated delayed fluorescence (TADF) dye 4CZIPN (PC), the excited state system is complex, comprising an initial prompt fluorescent state that converts an initial singlet state to an equilibrium mixed state of triplet and singlet. ${ }^{32-34}$ We studied the photocatalytic system using microsecond transient absorption spectroscopy (TAS). Our attention was focussed on two specific wavelengths: $420 \mathrm{~nm}$ and $810 \mathrm{~nm}$. At the former, a ground-state bleach is seen (see $\mathrm{SI}$ ), whereas at the latter a transient signal is observed (see $\mathrm{SI}$ ) that we assigned to absorbance of the ${ }^{3} \mathrm{CT}$-band of the photocatalyst as reported previously. ${ }^{32}$ The decay-constants $\left(\tau_{0}=3.89 \pm 0.05 \mu \mathrm{s}\right.$ ) of these signals matches the decay of the TADF observed by time-correlated single photon counting (see SI). Interestingly, upon addition of $\mathrm{HEH}$, the signal at $420 \mathrm{~nm}$ shows the formation of a transient species that subsequently vanishes $(\tau=8.2 \mu \mathrm{s}$, see Figure 3a red trace indicated by the arrow and SI), while at $810 \mathrm{~nm}$ we only observed quenching of the ${ }^{3} \mathrm{CT}$-band $(\tau=0.99 \pm 0.02 \mu \mathrm{s}$, see SI). The transient species is assigned to a charge-separated triplet cage-complex formed from the initial photoproducts: $\mathrm{PC}^{{ }^{--}}$and $\mathrm{HEH}^{{ }^{*}}$. Due to the charged nature of these species, they remain in close proximity as a cage complex, ${ }^{3}\left[\mathrm{PC}^{{ }^{--}}: \mathrm{HEH}^{\circ+}\right]$, and eventually recombine to reform PC and HEH without any observable permanent charge transfer (i.e., cage escape). The same experiment performed in the presence of collidine instead shows an irreversible transfer of electrons from HEH to PC, 
without recovery of the ground state (Figure $3 \mathrm{~b}$ red trace). It is clear that the base therefore prevents charge recombination by deprotonation of the ${ }^{3}\left[\mathrm{PC}^{\bullet-}: \mathrm{HEH}^{\bullet+}\right]$ intermediate. It is often assumed that the $\mathrm{HEH}^{\bullet+}$-species is highly acidic and will lose a proton rapidly; here we show that this is not generally the case, and hence the reason why photoredox catalytic reactions using $\mathrm{HEH}$, or similar reductive quenchers, often require (sub)stoichiometric amounts of base in order to provide productive process turnover. ${ }^{9,35-38}$

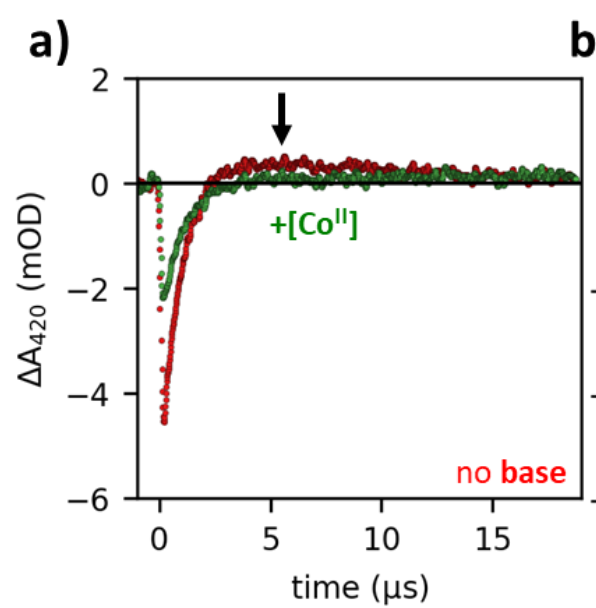

c)

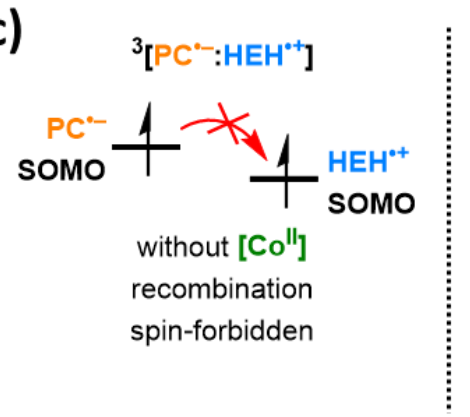

b)
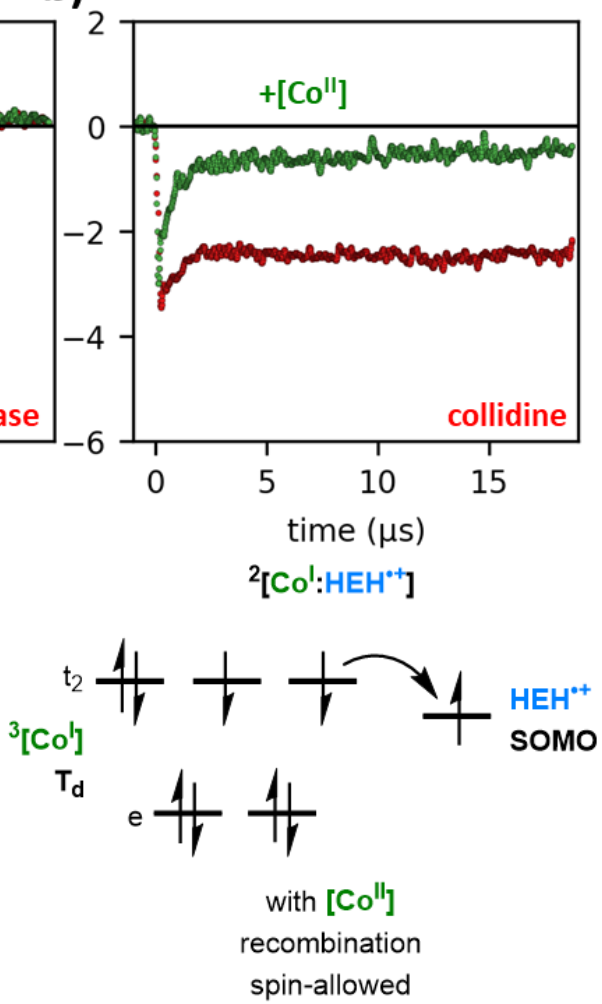

Figure 3. a) Transient absorption spectroscopy traces at $420 \mathrm{~nm}$. PC (50 $\mu \mathrm{M})$, HEH (200 $\mu \mathrm{M})$ without (red) or with (green) [Co"] $(170 \mu \mathrm{M})$. The arrow indicates the observed transient absorption signal assigned to ${ }^{3}\left[\mathrm{PC}^{--}: \mathrm{HEH}^{\bullet+}\right]$. b) like a but in the additional presence of 2,4,6-collidine $(50$ $\mathrm{mM}$ ). c) simplified $\mathrm{MO}$ diagram showing that charge recombination from the ${ }^{3}\left[\mathrm{PC}^{\circ-}: \mathrm{HEH}^{\bullet+}\right]$ is spin forbidden, but that after electron transfer to [Co"], fast charge recombination can occur to yield an excited-state ${ }^{2}\left[\mathrm{Co}^{\prime \prime}\right]$ that subsequently relaxes to the ground-state ${ }^{4}\left[\mathrm{Co}{ }^{\prime \prime}\right]$. SOMO $=\mathrm{Singly}$ occupied molecular orbital. $\mathrm{Td}$ = ligand field splitting in a tetrahedral environment.

The competition between the base and [Co"] in the kinetics, led us to hypothesise that the charge-separated state ${ }^{3}\left[\mathrm{PC}^{--}: \mathrm{HEH}^{\bullet+}\right]$ could also react with [Co"]. The traces at $420 \mathrm{~nm}$ of a mixture of PC and HEH were compared in the presence and absence of low concentrations of [Co"]. In the presence of the cobalt complex, the charge separated state ${ }^{3}\left[\mathrm{PC}^{--}: \mathrm{HEH}^{\cdot+}\right]$ is no longer observed (Figure 1e left), indicating a fast reaction with this state. No significant change in the decay of the TA-signal at $810 \mathrm{~nm}$ is observed (see $\mathrm{SI}$ ), therefore excluding the possibility of a reaction between the excited state ${ }^{3} \mathrm{PC}$ with the cobalt complex prior to the formation of ${ }^{3}\left[\mathrm{PC}^{\bullet-}: \mathrm{HEH}^{{ }^{*+}}\right]$. When collidine is also present in the sample, less permanent electron transfer is observed than in the absence of [Co"], showing that the reaction of ${ }^{3}\left[\mathrm{PC}^{\circ-}: \mathrm{HEH}^{\circ+}\right]$ with the cobalt complex is detrimental to the overall productivity of the reaction in agreement with the observed kinetics (Figure 2e and f). We propose that the complex reacts with the charge separated state to form a transient ${ }^{2}\left[\mathrm{Co}^{\prime}: \mathrm{HEH}^{\bullet+}\right]$-complex which leads to quantitative charge recombination. The reason that this cage-complex leads to fast charge-recombination is that recombination can occur through a spin-allowed process to produce an excited-state cobalt(II) complex, removing the need for a spin-flip (Figure 3c). DFT calculations on [Co"] indeed show that the excited (doublet) structure is only $1.1 \mathrm{eV}\left(26 \mathrm{kcal} \mathrm{mol}^{-1}\right)$ in energy above the ground-state (quartet) structure 
( $c f$ the energy of the photons of $2.8 \mathrm{eV}$ ), which is further confirmed by the presence of absorption peaks in the NIR of the $\left[\mathrm{Co}^{\prime \prime}\right]$-spectrum (Figure S14). Such low-lying metal excited states may limit the quantum efficiency in metallaphotoredox chemistry involving first-row transition metals by facilitating charge recombination, reminiscent of the ultrafast deactivation of metal-to-ligand charge-transfer (MLCT) excited states in first-row transition metal complexes compared to second- and third-row counterparts. ${ }^{39-41}$

Reduction of cobalt. Having these results in hand, the reduction of [CO"] was studied in detail. A cyclic voltammogram (CV) of the complex shows a reduction-peak at $E_{p}=-1.3 \vee v s \mathrm{Fc}^{+} \mid \mathrm{Fc}$ (Figure 4a left), which can be easily reduced by $\mathrm{PC}^{\cdot-}\left(E_{1 / 2}=-1.63 \vee v s \mathrm{Fc}^{+} \mid \mathrm{Fc}\right)$. The reduction is electrochemically irreversible, but the initial [Co"]-complex can be regenerated by applying more positive potentials (reoxidation peak at $E_{p}=-0.75 \mathrm{~V}$ ). Upon reduction to [Co'], the complex likely rapidly loses a $\mathrm{Cl}^{-}$ligand (at least up to $0.5 \mathrm{~V} \mathrm{~s}^{-1}$ no direct reoxidation peak is observed) that is then replaced either by solvent, ${ }^{42,43}$ or the complex dimerises to [Co(DPEPhos) $\left.(\mu-\mathrm{Cl})\right]_{2}{ }^{20,43}$ Spectroelectrochemistry confirmed the chemically reversible nature of the process, as the initial characteristic spectrum of [Co(DPEPhos) $\mathrm{Cl}_{2}$ ] can be recovered after a full CV (see SI). After the reduction wave, the spectrum shows an altered structure in the 550-800 $\mathrm{nm}$ range in addition to an intense band appearing at $390 \mathrm{~nm}$, which was assigned to a [Co']-complex. ${ }^{43}$ Interestingly, if the $\mathrm{CV}$ is measured in the presence of $\mathbf{1}$, the reduction wave becomes completely irreversible at slow scan-rates, indicating that $\left[\mathrm{Co}^{\prime}\right]$ is responsible for the oxidative addition of $\mathbf{1}$ to form a [Co'l-allyl] species (Figure $4 a$ right). At faster scan-rates, the reoxidation-peak reappears, allowing the determination of the rate-constant of oxidative addition, $k_{o a}=1.0 \mathrm{M}^{-1} \mathrm{~s}^{-1}$ (see $\mathrm{SI}$ ). The characteristic UV-vis spectrum of the species after oxidative addition was also obtained by spectroelectrochemistry, which looks very similar to that of [Co']. 


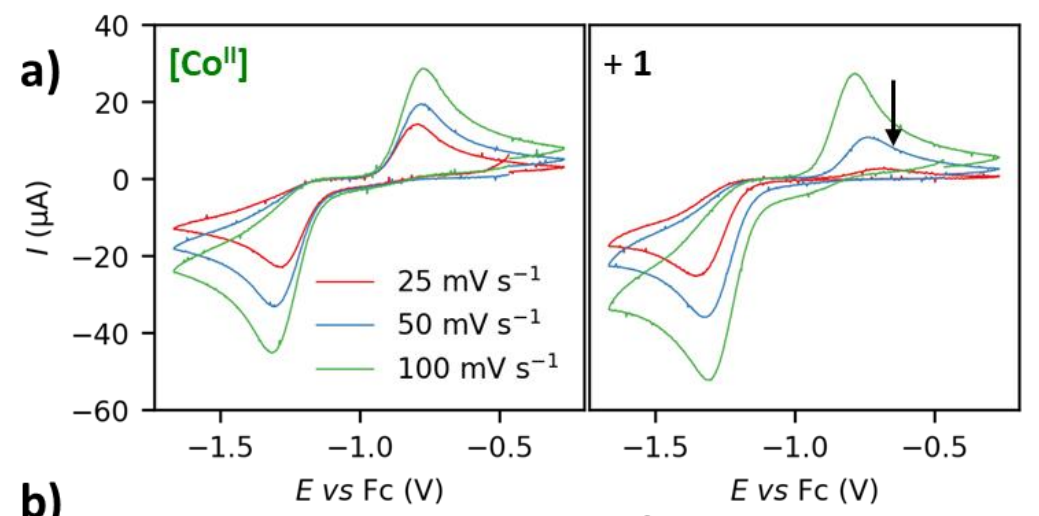

b)
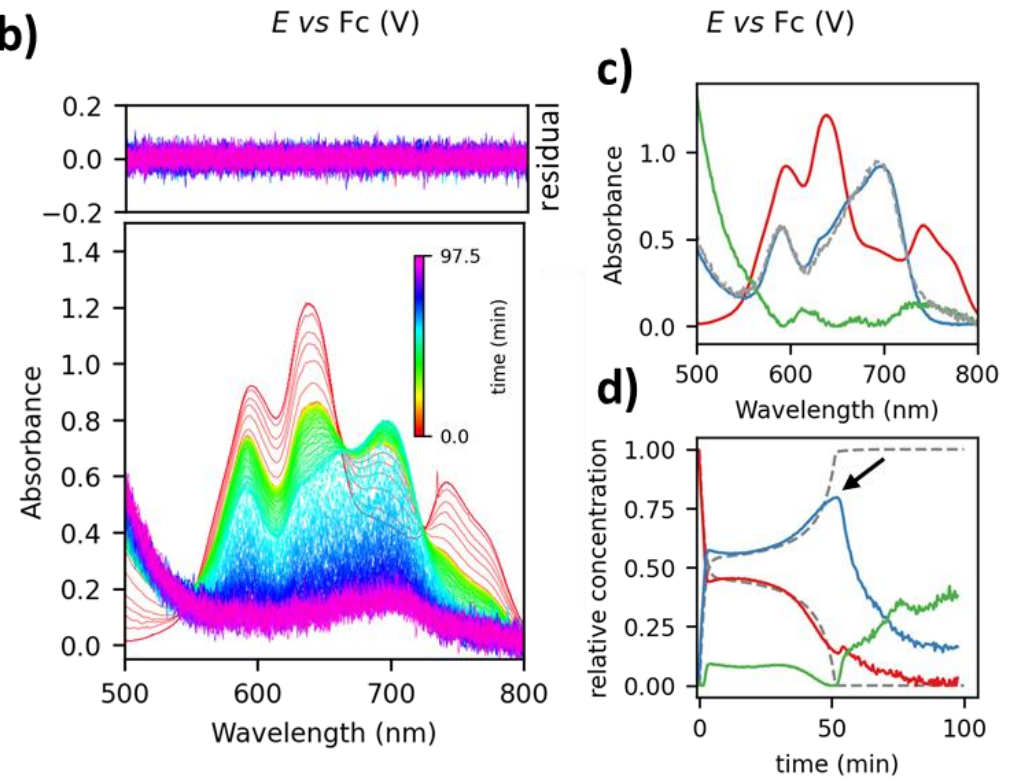

Figure 4. a) Cyclic voltammograms of [Co"] $(5 \mathrm{mM})$ in the absence (left) and presence (right) of $\mathbf{1}$ (50 mM). b) Evolution of the UV-vis spectrum under turnover conditions: $1(0.1 \mathrm{mmol}), 2(0.15 \mathrm{mmol}), \mathrm{HEH}(0.15 \mathrm{mmol})$, [Co"l] $(10 \mu \mathrm{mol}), \mathrm{PC}(2 \mu \mathrm{mol})$, collidine $(0.15 \mathrm{mmol}), \mathrm{THF}(3 \mathrm{~mL})$, blue LED irradiation $\left(\lambda_{\mathrm{em}}=445 \mathrm{~nm}, 0.1 \mathrm{~A}, 0.2 \mu\right.$ einstein $\left.\mathrm{s}^{-1}\right)$, and residual after a multivariate curve resolution fit using three spectral species. c) Spectral species obtained from multivariate curve fit of the UV-vis data. As a reference, the spectrum obtained by spectroelectrochemistry in the presence of 1 is plotted as a dashed grey line. d) Concentration profile of the spectral species in c (colours matching). The predicted cobalt species concentrations from the kinetic model are indicated as a dashed grey line (see SI for details). The arrow indicates the moment the spectra become noisy due to the precipitation of black metallic cobalt, coinciding with the moment the experiment and model start to differ significantly due to exhaustion of 1.

Using in situ UV-vis spectroscopy, we then monitored the spectral changes occurring under irradiation. In the absence of substrates $\mathbf{1}$ and 2, but in the presence of $\mathrm{HEH}$ and collidine, a fast conversion was observed to a species that resembles the spectrum obtained for [Co'] by spectroelectrochemistry (see SI). After this species is formed, further reduction occurs that leads to precipitation of a black magnetic solid that we assigned to metallic cobalt. In the absence of collidine, barely any changes in the UV-vis spectrum are observed, confirming the results obtained by TAS, showing the requirement and key role of the base. Next, the spectrum was monitored under turnover conditions (in the presence of $\mathbf{1}$ and $\mathbf{2}$ and collidine, see Figure $\mathbf{4 b}$ ). Rapidly a photostationary state is formed consisting of mainly 2 species: [Co"] and an intermediate species, that matches the [Co'l'-allyl] spectrum obtained from spectroelectrochemistry in the presence of 1 ( $c f$ the blue to the dashed grey spectrum in Figure $4 c$ ), although small contributions of [ $\left.\mathrm{Co}^{\prime}\right]$ are likely (see $\mathrm{SI}$ ), as the spectra might not be distinct enough. A third species (green curve), which we assign to $\left[\mathrm{Co}^{0}\right]$, becomes relevant only when the reaction is complete. The photostationary state evolves with the intermediate species becoming more pronounced until substrate 1 is exhausted (Figure $4 \mathrm{~d}$ ), after which precipitation of metallic cobalt occurs (indicated by the arrow in Figure 4d). These observations indicate a fast catalytic cycle that is limited by the production of reductive equivalents through photoinduced electron transfer. After a further 
reduction of [Co'l'-allyl] to [Co"-allyl], the formation of product and regeneration of [Co"] is fast (vide infra). As such, the cobalt catalyst is a mixture of mainly two resting states, viz. [Co'l'-allyl] and the starting complex [Co"], that both await further photoinduced reduction. As substrate $\mathbf{1}$ is nearing exhaustion, the rate of oxidative addition decreases, leading to an increase in [Co']. Satisfyingly, the fitted kinetic model (vide infra and SI), shown by the dashed grey curves in Figure $4 \mathrm{~d}$ captures the evolution of cobalt species during the reaction remarkably well when the intermediate species is assumed to be the sum of [Co'l'-allyl] and [Co'], whose spectra are hard to distinguish (see SI).

Product formation. Density functional theory (B3LYP-D3 in THF solvent, see SI for full details) was employed to elucidate the final steps of the mechanism starting from [CO"-allyl] B and explain the formation of the 1,3-diol $\mathbf{3}$ in a high 95:5 syn:anti ratio. Three different key steps beyond the [Co"-allyl] intermediate B were considered: isomerisation of the allylic moiety (i.e, $\mathbf{B}_{\mathbf{Z}}$ to $\mathrm{B}_{\mathrm{E}}$ and vice versa), $\mathrm{C}-\mathrm{C}$ bond formation and a protodemetalation stage. For the $\mathrm{C}-\mathrm{C}$ bond formation, we optimised the pathway through well-known Zimmerman-Traxler-type transition states (TSs). In principle, four different stereoisomeric pathways can exist upon coordination of substrate 2 . The allyl species could have either an $E$ or $Z$ configuration, and substrate $\mathbf{2}$ could be positioned pseudo-equatorial of pseudo-axial. However, due to the steric nature of the DPEPhos ligand, we excluded the possibility of a pseudo-axial positioning of substrate 2 due to unfavourable steric 1,3-diaxial interactions. ${ }^{44}$ Therefore, only the pathways using either $E$ or $Z$ isomers of the [Co"-allyl] were considered. The overall barriers for C-C bond formation are low (15.9 and $17.8 \mathrm{kcal} \mathrm{mol}^{-1}$ for forming the syn and anti-product, respectively), in agreement with a fast reaction that was observed experimentally. The protodemetalation step for the anti-isomer (see SI) presents a slightly higher barrier than for the preceding C-C bond formation step, but this seems a minor computational artefact, as only minimal changes in $d r$ were observed experimentally when comparing different bases (see SI for discussion). ${ }^{26}$

On the basis of the energies of the simplified path (Figure 5), we therefore conclude that the stereochemistry is determined by differences in the steric requirements of the $E$ and Z-allylic isomeric forms of the Zimmerman-Traxler TS. Isomerisation of the allyl is comparable in rate to $\mathrm{C}-\mathrm{C}$ bond formation, allowing interconversion of the $E$ to the $Z$ allyl species to give a higher ratio of the syn product. The diastereoselectivity predicted from microkinetic modelling (96:4 $d r$, see SI) aligns well with the experimental value (95:5 $d r$ ), supporting the view that the Zimmerman-Traxler TS determines the stereoselective outcome of the transformation. It should be noted that the energies of the three steps are close enough that small alterations to the system might lead to a different stereodetermining step, and therefore a large difference in $d r$. Indeed, the ligand, substrate and base have all shown to influence the $d r$ in varying degrees. ${ }^{26}$ 

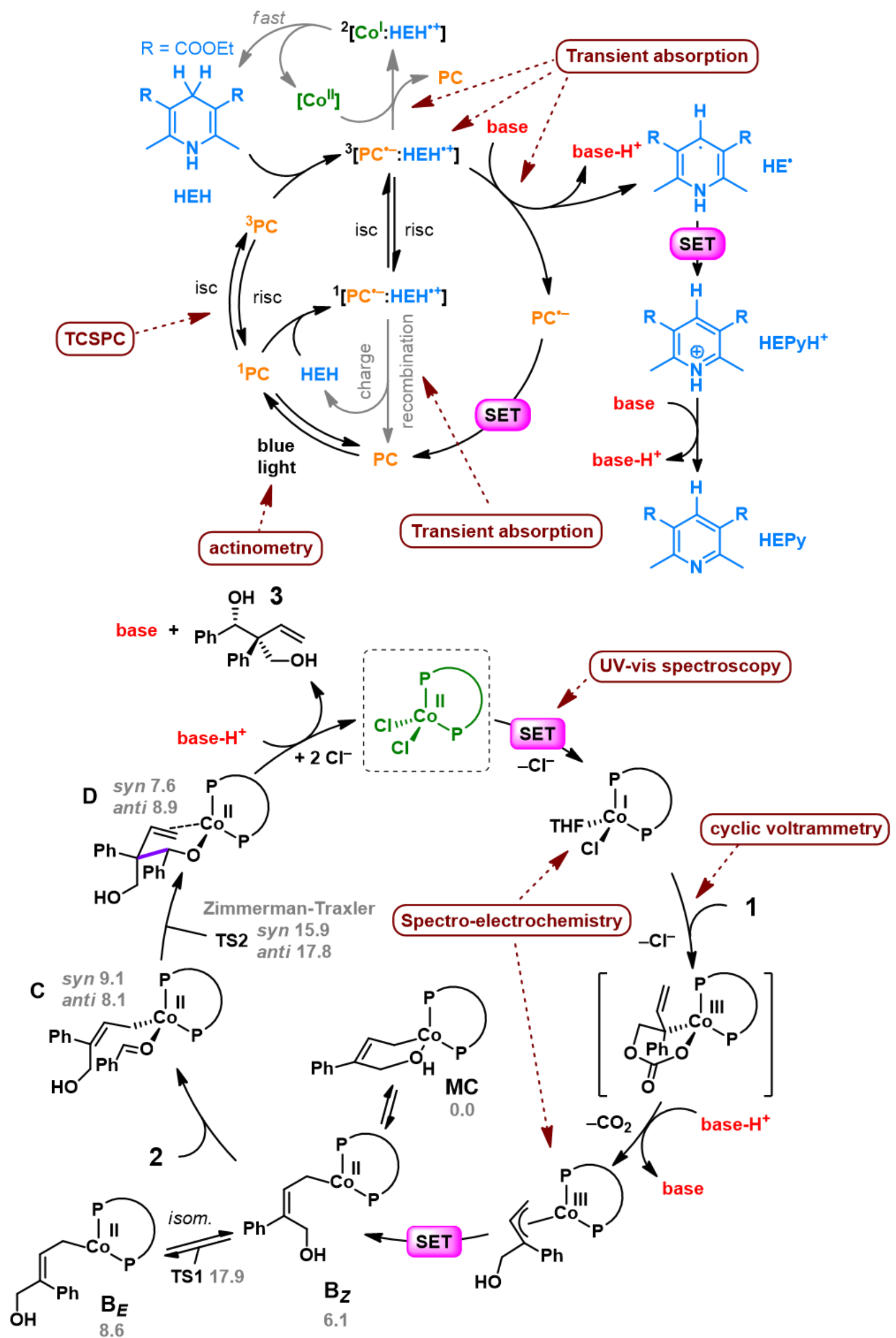

Figure 5. Full elucidated mechanism of the photoredox cycle (top) and cobalt cycle (bottom) with DFT energies (kcal / mol) in grey for selected steps. Techniques used to observe various steps in the mechanism are indicated. Only the pathway from the $Z$ isomer towards the major product (syn) is shown; a corresponding higher energy pathway exists for the $E$-allyl isomer. 
Kinetic modelling. Combining all observations and measured rate constants, an overall mechanism and kinetic model was proposed (Figure 5 with detailed information in the SI). The known photophysical reactions were used and an additional charge-separated state $\left({ }^{1}\left[\mathrm{PC}^{\bullet-}: \mathrm{HEH}^{\bullet+}\right]\right)$ was introduced that is formed by reductive quenching of the singlet state, which is a prevalent pathway under turnover conditions. This species can undergo fast inter-system crossing to

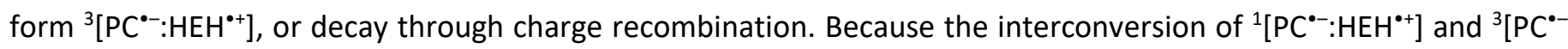
$: \mathrm{HEH}^{\left.{ }^{+}\right]}$was not observed by TAS, they are expected to be much faster than the subsequent reactions from the ${ }^{3}\left[\mathrm{PC}^{\cdot-}\right.$ $\left.: \mathrm{HEH}^{{ }^{+}}\right]-$complex, and therefore the ratio of ${ }^{3}\left[\mathrm{PC}^{--}: \mathrm{HEH}^{{ }^{+}}\right]$and ${ }^{1}\left[\mathrm{PC}^{--}: \mathrm{HEH}^{\circ+}\right]$ are in preequilibrium. We therefore used the yield of intersystem crossing from the singlet state (i.e., the part of ${ }^{1}\left[\mathrm{PC}^{--}: \mathrm{HEH}^{{ }^{+}}\right]$reacting through isc vs charge recombination) as a fitting constant. Other unknown parameters were the rates of deprotonation by the three bases (i.e., $\mathrm{K}_{3} \mathrm{PO}_{4}, \mathrm{HEPy}$ or collidine), as well as the rate of deactivation of ${ }^{3}\left[\mathrm{PC}^{--}: \mathrm{HEH}^{{ }^{+}}\right]$with $\left[\mathrm{Co}(\mathrm{DPEPhos}) \mathrm{Cl}_{2}\right]$, which was too fast to be observed by TAS. All other unknown rate constants did not alter the overall process as long as they were sufficiently high. A global fit was performed on the in situ IR data, where all the rates were shared as parameters (see $\mathrm{SI}$ ). The excellent fit to the experimental data (see Figure $1 \mathrm{~b}$, d, Figure $4 \mathrm{~d}$ and $\mathrm{SI}$ ) shows that the model can be validated as it well captures the important parts of the reaction path, and predicts the intrinsic bottlenecks. Importantly, the interactions in the initial charge-separated state with either base or transition metal catalyst are key for the efficiency of the reaction. Indeed, many of the absorbed photons are 'wasted' due to recombination processes from the ${ }^{3}\left[\mathrm{PC}^{\circ-}\right.$ $\left.: \mathrm{HEH}^{\circ+}\right]$-state. Such interactions are likely to be prevalent in many other metallaphotoredox catalysis processes, and consequently would lead to unproductive pathways especially when low-lying excited states are available on the transition metal catalyst.

\section{Conclusion}

In summary, key inter-catalyst reactions determine the efficiency of this representative cross-electrophile C-C bond formation reaction. The base deprotonates the photochemically-generated transient charge-separated state, preventing rapid charge recombination that occurs in absence of the base and thus enabling further electron transfer. The byproduct of the reaction, HEPy, is itself a base, and therefore the reaction follows an autocatalytic kinetic profile. We further show that the cobalt complex reacts competitively with the transient charge-separated state, leading to catalyst-induced charge recombination and therefore an unintuitive negative order in [Co"]. Such counterproductive interactions are expected to be generally present in reductive metallaphotoredox catalytic reactions employing $3 \mathrm{~d}$ metals as charge recombination in cage-complexes might be facilitated by low-lying excited states of the transitionmetal complex. These intricacies occurring within the SET step can easily inhibit reactions, and therefore should be considered when metallaphotoredox promoted reactions are investigated. Elucidation of the underlying mechanistic complexity of multi-catalyst systems will help to further advance the field of metallaphotoredox chemistry as a powerful tool to uncover new transformations in synthetic chemistry.

\section{Acknowledgments}

Prof. Feliu Maseras is thanked for theoretical chemistry advice and fruitful scientific discussion. Raúl Pérez Soto is thanked for help with setting up DFT calculations. Dr. Javier Pérez is thanked for help with transient absorption 
spectroscopy. Dr. Marcos Gil Sepulcre and Prof. Antoni Llobet are thanked for help with spectroelectrochemistry. This project has received funding from the European Union's Horizon 2020 research and innovation programme under grant agreement No. 889754, and from MICINN (PID2020-112684GB-100 and Severo Ochoa Excellence Accreditation 2020-2023 CEX2019-000925-S). We also acknowledge the Cerca program/Generalitat de Catalunya and ICREA for support.

\section{Author contributions}

BL performed all experimental work presented in the study. BL performed DFT calculations. BL wrote python scripts and performed modelling of the data. AC performed preliminary control experiments. BL and AC interpreted the data. $B L$ designed the study. BL, AC and AK drafted and revised the manuscript. AK provided feedback and contextualisation of the work.

\section{References}

1. Twilton, J. et al. The merger of transition metal and photocatalysis. Nat. Rev. Chem. 1, Article number: 0052 (2017).

2. Wang, W.-Z. et al. Light-Driven Molecular Motors Boost the Selective Transport of Alkali Metal lons through Phospholipid Bilayers. J. Am. Chem. Soc. 143, 15653-15660 (2021).

3. Gualandi, A. et al. Metallaphotoredox catalysis with organic dyes. Org. Biomol. Chem. 19, 3527-3550 (2021).

4. Charboneau, D.J., Barth, E.L., Hazari, N., Uehling, M.R. \& Zultanski, S.L. A Widely Applicable Dual Catalytic System for Cross-Electrophile Coupling Enabled by Mechanistic Studies. ACS Catal. 142, 12642-12656 (2020).

5. Sakai, H.A., Liu, W., Le, C. \& MacMillan, D.W.C. Cross-Electrophile Coupling of Unactivated Alkyl Chlorides. J. Am. Chem. Soc. 142, 11691-11697 (2020).

6. Kerackian, T., Reina, A., Bouyssi, D., Monteiro, N. \& Amgoune, A. Silyl Radical Mediated Cross-Electrophile Coupling of N-Acyl-imides with Alkyl Bromides under Photoredox/Nickel Dual Catalysis. Org. Lett. 22, 22402245 (2020).

7. Everson, D.A. \& Weix, D.J. Cross-electrophile coupling: Principles of reactivity and selectivity. Journal of Organic Chemistry vol. 79 4793-4798 (2014).

8. Kojima, M. \& Matsunaga, S. The Merger of Photoredox and Cobalt Catalysis. Trends Chem. 2, 410-426 (2020).

9. Li, Y.-L., Zhang, S.-Q., Chen, J. \& Xia, J.-B. Highly Regio- and Enantioselective Reductive Coupling of Alkynes and Aldehydes via Photoredox Cobalt Dual Catalysis. J. Am. Chem. Soc. 143, 7306-7313 (2021).

10. Yasui, T., Yamada, K., Tatsumi, R. \& Yamamoto, Y. Cobalt/Organophotoredox Dual-Catalysis-Enabled Cascade Cyclization of 1,6-Diynyl Esters via Formal 1,8-Acyloxy Migration. ACS Catal. 11, 11716-11722 (2021).

11. Ma, W.-Y. et al. Cobalt-Catalyzed Enantiospecific Dynamic Kinetic Cross-Electrophile Vinylation of Allylic Alcohols with Vinyl Triflates. J. Am. Chem. Soc. 143, 15930-15935 (2021).

12. Till, N.A., Tian, L., Dong, Z., Scholes, G.D. \& MacMillan, D.W.C. Mechanistic Analysis of Metallaphotoredox C$\mathrm{N}$ Coupling: Photocatalysis Initiates and Perpetuates Ni(I)/Ni(III) Coupling Activity. J. Am. Chem. Soc. 142, 15830-15841 (2020).

13. Qin, Y. et al. Mechanistic Investigation and Optimization of Photoredox Anti-Markovnikov Hydroamination. J. Am. Chem. Soc. 143, 10232-10242 (2021).

14. Sun, R. et al. Elucidation of a Redox-Mediated Reaction Cycle for Nickel-Catalyzed Cross Coupling. J. Am. Chem. Soc. 141, 89-93 (2018).

15. Tian, L., Till, N.A., Kudisch, B., MacMillan, D.W.C. \& Scholes, G.D. Transient Absorption Spectroscopy Offers Mechanistic Insights for an Iridium/Nickel-Catalyzed C-O Coupling. J. Am. Chem. Soc. 142, 4555-4559 (2020).

16. Qin, Y., Sun, R., Gianoulis, N.P. \& Nocera, D.G. Photoredox Nickel-Catalyzed C-S Cross-Coupling: Mechanism, Kinetics, and Generalization. J. Am. Chem. Soc. 143, 2005-2015 (2021).

17. Ma, Y. \& Han, Z. Computation Revealed Mechanistic Complexity of Low-Valent Cobalt-Catalyzed Markovnikov Hydrosilylation. J. Org. Chem. 83, 14646-14657 (2018).

18. Oliveira, J.C.A., Dhawa, U. \& Ackermann, L. Insights into the Mechanism of Low-Valent Cobalt-Catalyzed C-H Activation. ACS Catal. 11, 1505-1515 (2021). 
19. Dorval, C., Tricoire, M., Begouin, J.-M., Gandon, V. \& Gosmini, C. Cobalt-Catalyzed C(sp2)-CN Bond Activation: Cross-Electrophile Coupling for Biaryl Formation and Mechanistic Insight. ACS Catal. 10, 12819-12827 (2020).

20. Friedfeld, M.R., Zhong, H., Ruck, R.T., Shevlin, M. \& Chirik, P.J. Cobalt-catalyzed asymmetric hydrogenation of enamides enabled by single-electron reduction. Science 360, 888-893 (2018).

21. Gray, M. et al. Mechanism of Cobalt-Catalyzed Heterodimerization of Acrylates and 1,3-Dienes. A Potential Role of Cationic Cobalt(I) Intermediates. ACS Catal. 10, 4337-4348 (2020).

22. Hickey, D.P. et al. Investigating the Role of Ligand Electronics on Stabilizing Electrocatalytically Relevant LowValent Co(I) Intermediates. J. Am. Chem. Soc. 141, 1382-1392 (2019).

23. Wang, L., Wang, L., Li, M., Chong, Q. \& Meng, F. Cobalt-Catalyzed Diastereo- and Enantioselective Reductive Allyl Additions to Aldehydes with Allylic Alcohol Derivatives via Allyl Radical Intermediates. J. Am. Chem. Soc. 143, 12755-12765 (2021).

24. Gualandi, A. et al. Catalytic Photoredox Allylation of Aldehydes Promoted by a Cobalt Complex. Adv. Synth. Catal. 363, 1105-1111 (2020).

25. Shi, C. et al. Photocatalytic Umpolung Synthesis of Nucleophilic $\pi$-Allylcobalt Complexes for Allylation of Aldehydes. ACS Catal. 11, 2992-2998 (2021).

26. Cristòfol, À., Limburg, B. \& Kleij, A.W. Expedient Dual Co/Organophotoredox Catalyzed Stereoselective Synthesis of All-Carbon Quaternary Centers. Angew. Chem. Int. Ed. 60, 15266-15270 (2021).

27. Schwarz, J.L., Schäfers, F., Tlahuext-Aca, A., Lückemeier, L. \& Glorius, F. Diastereoselective Allylation of Aldehydes by Dual Photoredox and Chromium Catalysis. J. Am. Chem. Soc. 140, 12705-12709 (2018).

28. Gualandi, A. et al. Cp2TiCl2-Catalyzed Photoredox Allylation of Aldehydes with Visible Light. ACS Catal. 10, 3857-3863 (2020).

29. Mitsunuma, H., Tanabe, S., Fuse, H., Ohkubo, K. \& Kanai, M. Catalytic asymmetric allylation of aldehydes with alkenes through allylic $\mathrm{C}(\mathrm{sp} 3)-\mathrm{H}$ functionalization mediated by organophotoredox and chiral chromium hybrid catalysis. Chem. Sci. 10, 3459-3465 (2019).

30. Tanabe, S., Mitsunuma, H. \& Kanai, M. Catalytic Allylation of Aldehydes Using Unactivated Alkenes. J. Am. Chem. Soc. 142, 12374-12381 (2020).

31. Gualandi, A. et al. Allylation of aldehydes by dual photoredox and nickel catalysis. Chem. Commun. 55, 68386841 (2019).

32. Noda, H. et al. Critical role of intermediate electronic states for spin-flip processes in charge-transfer-type organic molecules with multiple donors and acceptors. Nat. Mater. 18, 1084-1090 (2019).

33. Kobayashi, T. et al. Intersystem Crossing Rate in Thermally Activated Delayed Fluorescence Emitters. Phys. status solidi 217, 1900616 (2020).

34. Ishimatsu, R. et al. Solvent effect on thermally activated delayed fluorescence by 1,2,3,5-tetrakis(carbazol-9yl)-4,6-dicyanobenzene. J. Phys. Chem. A 117, 5607-5612 (2013).

35. Meng, Q.Y., Schirmer, T.E., Katou, K. \& König, B. Controllable Isomerization of Alkenes by Dual Visible-LightCobalt Catalysis. Angew. Chem. Int. Ed. 58, 5723-5728 (2019).

36. Meng, Q.Y., Wang, S., Huff, G.S. \& Konig, B. Ligand-Controlled Regioselective Hydrocarboxylation of Styrenes with $\mathrm{CO} 2$ by Combining Visible Light and Nickel Catalysis. J. Am. Chem. Soc. 140, 3198-3201 (2018).

37. Meng, Q.Y., Wang, S. \& König, B. Carboxylation of Aromatic and Aliphatic Bromides and Triflates with $\mathrm{CO} 2$ by Dual Visible-Light-Nickel Catalysis. Angew. Chem. Int. Ed. 56, 13426-13430 (2017).

38. Wang, P.Z., Chen, J.R. \& Xiao, W.J. Hantzsch esters: An emerging versatile class of reagents in photoredox catalyzed organic synthesis. Org. Biomol. Chem. 17, 6936-6951 (2019).

39. Auböck, G. \& Chergui, M. Sub-50-fs photoinduced spin crossover in [Fe(bpy)3]2+. Nat. Chem. 2015 78 7, 629633 (2015).

40. Förster, C. \& Heinze, K. Photophysics and photochemistry with Earth-abundant metals - fundamentals and concepts. Chem. Soc. Rev. 49, 1057-1070 (2020).

41. Wegeberg, C. \& Wenger, O.S. Luminescent First-Row Transition Metal Complexes. JACS Au 1, 1860-1876 (2021).

42. Lindner, E., Schober, U., Glaser, E., Norz, H. \& Wegner, P. Neuartige basische Liganden für die homogenkatalytische Methanolcarbonylierung, XI [1] Ether-Phosphan-Cobalt-Komplexe in der katalytischen Methanolhydrocarbonylierung zu Acetaldehyd. Zeitschrift fur Naturforsch. - Sect. B J. Chem. Sci. 42, 15271536 (1987).

43. Aresta, M., Rossi, M. \& Sacco, A. Tetrahedral complexes of cobalt(I). Inorganica Chim. Acta 3, 227-231 (1969).

44. Mejuch, T. et al. Axial preferences in allylation reactions via the Zimmerman-Traxler transition state. Acc.

Chem. Res. 46, 1659-1669 (2013). 
